\title{
Water Situation in Qingdao
}

\author{
Maowei Zhu ${ }^{1, \text { a }}$ \\ ${ }^{1}$ School of the North China Electric Power University, Baoding 071000, China \\ a1076665449@qq.com
}

Keywords: Water situation the Fuzzy Synthetic Evaluation (FSE) the Partial Least Square Regression (RLSR)

\begin{abstract}
This paper mainly analyzes water situation in Qingdao. First, the Fuzzy Synthetic Evaluation (FSE) model is used to determine water shortage types. What's more, we build up the Partial Least Square Regression (RLSR) model to analyze social and environmental drivers for water scarcity in Qingdao. Furthermore, we apply Bayesian Network to build up a relationship between the WSD indicators and life quality indicators.
\end{abstract}

\section{Introduction}

Humans require water resources for industrial, agricultural, household, and environmental needs. And the water supply not only constrained by total water resources of a region, but also the water management. Water scarcity can be a result of two mechanisms: physical water scarcity and economic water scarcity, where physical water scarcity is the result of in adequate natural water supply a region's demand, and economic water scarcity is a result of poor management of the sufficient available water resources [1].

\section{Model I: Fuzzy Synthetic Evaluation}

In this section, we are committed to analyze the shortage types of Qingdao (physical water scarcity and/or economic water scarcity). And since the classification in this field is characterized by fuzziness, the fuzzy model is applicable and reasonable in this question.

a) Identify alternatives and attributes

Referring to the water resources rational allocation evaluation system, we choose per capita water resources (PCWR), water resources utilization (WRU), per capita water supply (PCWS), river water quality (RWQ) and water resource management (WRM) as our evaluation criteria to estimate water scarcity type of a region. According to the reference [2], we construct a criteria-evaluation table for two typical water scarcity types, as shown in Table 1.

Table 1 The criteria-evaluating table for two typical water scarcity types

\begin{tabular}{cccc}
\hline number & indicators & physical & economic \\
\hline 1 & per capita water resources (PCWR) & $\leq 1500$ & $>1500$ \\
2 & per capita water supply (PCWS) & $\geq 40 \%$ & $\leq 20 \%$ \\
3 & water resources utilization (WRU) & $\leq 500$ & $\leq 500$ \\
4 & river water quality (RWQ) & medium & medium \\
5 & water resource management (WRM) & fine & fine \\
\hline
\end{tabular}

b) Determine fuzzy evaluation matrix

According to Table 1, we conduct the normalizing process based on:

For per capita water resources (PCWR), the bigger the better, if $P C W R>1500$, then $r_{i j}=1$; otherwise $r_{i j}=0$; For per capita water supply (PCWS), the bigger the better, if $P C W S>500$, then $r_{i j}=1$; otherwise $r_{i j}=0$; For water resources utilization (WRU), the smaller the better, if $W R U \geq 40 \%$, then $r_{i j}=0$; if $20 \%<W R U<40 \%$, then $r_{i j}=0.5$; if $W R U \leq 20 \%$, then $r_{i j}=1$; For river water quality (RWQ) and water resource management (WRM), if RWQ (WRM) is good, 
then $r_{i j}=1$; if it is medium, then $r_{i j}=0.5$; if it is poor, then $r_{i j}=0$.

Therefore, we have the fuzzy evaluation matrix

$$
S=\left[\begin{array}{cc}
0 & 1 \\
0 & 1 \\
0 & 0 \\
0.5 & 0.5 \\
1 & 1
\end{array}\right]
$$

c) Determine the matrix of fuzzy characteristics

According to data collected from reference [3], the matrix of fuzzy characteristics is:

$$
r=\left[\begin{array}{lllll}
0 & 0 & 0 & 0 & 0.5
\end{array}\right]^{\mathrm{T}}
$$

d) Determine the subjection matrix

Using expert consultation method, we get the weight of each evaluation is the same, and then we normalized the weighting vector $w=\left[\begin{array}{lllll}0.2 & 0.2 & 0.2 & 0.2 & 0.2\end{array}\right]^{\mathrm{T}}$

Then we calculate the subjection matrix: $U=\left[\begin{array}{ll}0.73 & 0.27\end{array}\right]$

e) Results Analysis

We can see from equitation (4) that physical water scarcity is the main cause for water scarcity in Qingdao. Since the water resource distribution is not uniform because of geological and topographical reasons, which brings great difficulties to water utilize; Both less water resources engineering and poor water regulating ability make economic water scarcity is also a cause for water scarcity in Qingdao.

\section{Model II: the Partial Least Square Regression Model (PLSR)}

In this section, The PLCR method [2] is used to analyze drivers for water scarcity in Qingdao city. First, we select 16 indicators' data and water scarcity ratio of Qingdao in the past 8 years from Shandong Statistical Information Net [3]. These indicators are shown in below, which have been proved to be effective to reflect water situation of a region.

Table 2 Systems of indicators for water supply and water demand

\begin{tabular}{lll}
\hline & Level 1 & Level 2 \\
& & Annual precipitation \\
& Agricultural needs & Annual average temperature \\
& & Evapotranspiration \\
Water demand & Industrial needs & Sown area \\
& & Agricultural production \\
& & Industrial production \\
& Household needs & Urban population \\
& & Rural population \\
& & Urban income \\
& & Rural income \\
Total water resources & Green area \\
\hline \multirow{2}{*}{ Water supply } & & Surface water \\
& & Ground water \\
& & Reclaimed water \\
& & Industrial sewage \\
& & Domestic sewage \\
\hline
\end{tabular}

The collected data will be removed from the singular value while the individual omissions interpolation will be filled according to the data to make a total of eight year data. The data are 
divided into two groups, in which the former seven years data ( $1^{\text {st }}$ group) is to establish the model and the latter one year data ( $2^{\text {nd }}$ group) to test the model results.

The regression equation of water scarcity ratio (WSR) is

$$
\begin{aligned}
W S R= & -0.25 x_{1}-0.06 x_{2}+0.04 x_{3}+0.05 x_{4}+0.04 x_{5} \\
& +0.07 x_{6}+0.33 x_{7}-0.32 x_{8}+0.06 x_{9}+0.06 x_{10} \\
& +0.05 x_{11}-0.06 x_{12}-0.27 x_{13}-0.06 x_{14}+0.26 x_{15}+0.29 x_{16},
\end{aligned}
$$

where $x_{i}, i=1,2, \ldots, 16$ is the $i$ th indicator shown in Table 2.

The comparisons of fitting and collected values of water scarcity ratio are shown in Figure 1.

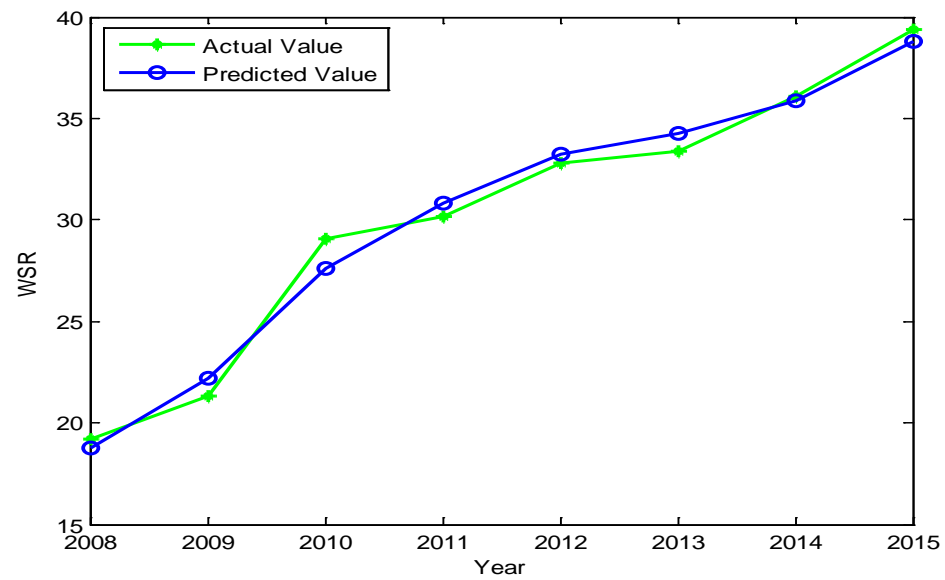

Figure 1 Comparison of fitting and collected values of water scarcity ratio

According to Figure 1, considering the influence of the data errors, the results of PLSR model has basically achieve the request of the precision.

By analyzing the equitation (11), we can find that domestic sewage, industrial sewage, annual precipitation, urban population, rural population, and reclaimed water are the main drivers of water scarcity in Qingdao city.

\section{Problems Tied to the Water Scarcity}

With the purpose of giving a full-scale evaluation of the lives of citizens, a series of indicators is used, which are shown in Table 3.

Table 3 the life quality evaluation system

\begin{tabular}{cc}
\hline Level 1 & Level 2 \\
\hline Economic level & per capita disposal income \\
Health condition & annual outpatients amount \\
& per capita green area \\
Environment & wastewater purification rate \\
\hline
\end{tabular}

How does water situation impact the lives of citizens of Qingdao? According to reference [4][5], we first find the relationship between our first-level indicators of the WSD model (water resources, water management, industrial, agricultural, household and environmental needs) and the second-level indicators of the life quality evaluation system (Table 3). Then we establish the regression relationship between them by applying Bayesian Network. Subsequently, the relationship between water scarcity and life quality is built up (Figure1), which can roundly reflect the water situation's impaction on the lives of citizens.

As a branch of machine learning in dealing with uncertain and complex interdependence between various factors in the system, the Bayesian Network has a unique strong type inference and visualization. Since in our problem, the life quality is fuzzy, and there are many direct or indirect reasons to influence live quality, so we choose the Bayesian Network in this section.

We use the annual outpatients' amount to illustrate our model. 


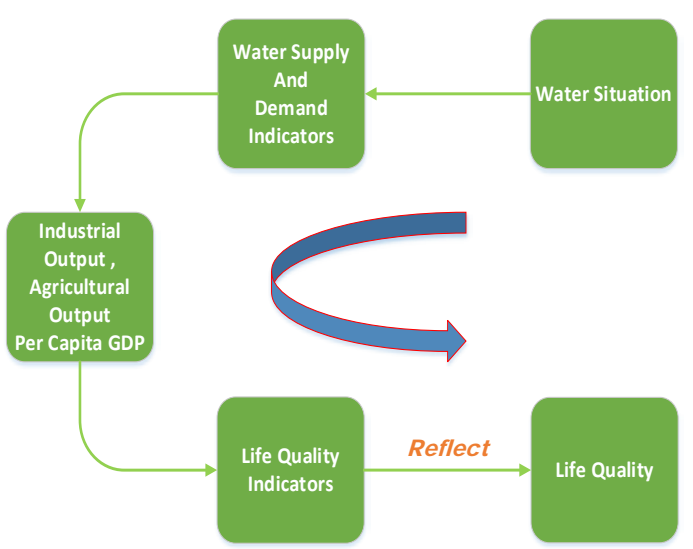

Figure 1 the relationship between water scarcity and life quality

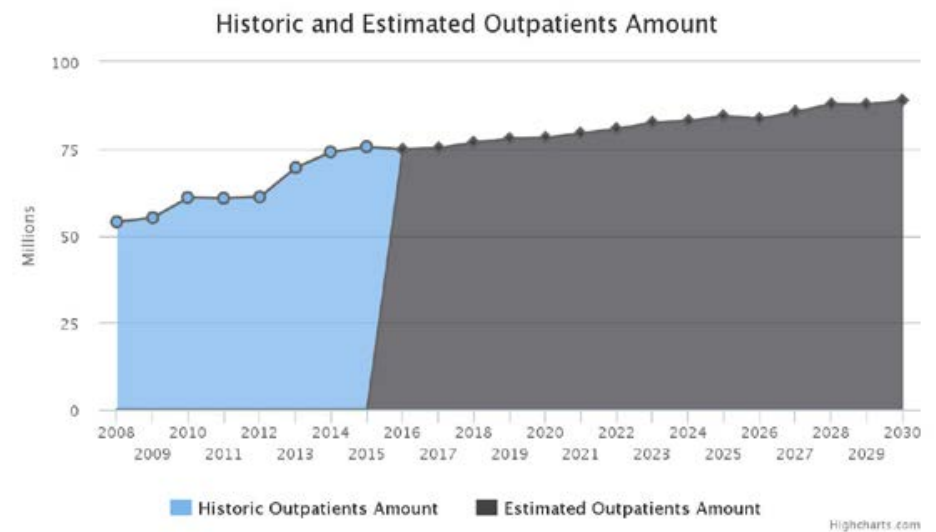

Figure 2 Historic and Estimated Outpatients' Amount

From Figure 2, we can find the annual outpatients' amount increases in the following 15years, which indicates that, the health condition of people is declining in the next 15years.

In the same way, we analyze other indicators' change, and find the impactions of water situation on lives of citizens of Qingdao are:

- $\quad$ per capita disposal income is nearly unchanged, which indicates the economic level of Qingdao is unchanged in the 15years

- annual outpatients' amount is creasing slowly, which indicates the decline of citizen's health condition

- $\quad$ per capita green area and wastewater purification rate are decreased slowly, which indicates that environment condition is becoming worse and worse.

\section{Conclusions}

By applying The Fuzzy Synthetic Evaluation (FSE) model, we find that physical water scarcity is the main cause for water scarcity in Qingdao. Since the water resource distribution is not uniform because of geological and topographical reasons, which brings great difficulties to water utilize. And both less water resources engineering and poor water regulating ability make economic water scarcity is also a cause for water scarcity in Qingdao. What's more, we build up the Partial Least Square Regression (RLSR) model to analyze social and environmental drivers for water scarcity, and finally find that domestic sewage, industrial sewage, annual precipitation, urban population, rural population, and reclaimed water are the main drivers of water scarcity in Qingdao.

\section{References}

[1] Wikipedia. Physical water scarcity [online]. Available from: https : // en. Wikipedia .org / wiki / Physical _ water _scarcity. [Accessed on 29 $9^{\text {th }}$ January, 2016].

[2] Luo, B, Zhao, Y, Chen, K., \& Zhao, X. (2009). Partial least squares regression model to predict water quality in urban water distribution systems. Transactions of Tianjin University, 15, 140-144.

[3] Shandong Statistical Information Net [online]. Available from: http : // www. Stats -sd. gov. Cn /col / col211 /index. html. [Accessed on 30 ${ }^{\text {th }}$ January, 2016].

[4] Ren Zhong, (2005). Water resources and sustainable economic development: use Dalian as an example. Master degree theses of master of northeast university of finance and economics.

[5] Arbués, F., Barberán, R., \& Villanúa, I. (2004). Price impact on urban residential water demand: a dynamic panel data approach. Water Resources Research, 40(11). 